\title{
Ciencia psicológica y profesionalización en Argentina y Brasil: 1930-1980*
}

\author{
Psychological Science and Professionalization \\ in Argentina and Brazil: 1930-1980
}

Recibido: enero 15 de 2014 | Revisado: julio 26 de 2014 | Aceptado: julio 27 de 2014

\author{
MARÍA ANDREA PIÑEDA ** \\ Consejo Nacional de Investigaciones Científicas \\ y Técnicas (CONICET) \\ Universidad Nacional de San Luis (UNSL), Argentina \\ ANA MARÍA JACÓ-VILELA **** \\ Universidade do Estado do Rio de Janeiro, Brasil
}

doi:10.11144/Javeriana.upsy13-5.cppa

Para citar este artículo: Piñeda, M. A., \& JacóVilela, A. M. (2014). Ciencia psicológica y profesionalización en Argentina y Brasil: 1930-1980. Universitas Psychologica, 13(5), 2015-2033. http:/ dx.doi.org/10.11144/Javeriana.upsy13-5.cppa

Financiamiento: 1- Proyecto: "Incidencia de publicaciones periódicas y colecciones editoriales de psicología en la formación del psicólogo en Argentina: 1954-1984", dirigido por el Dr. Hugo Klappenbach (CONICET/Universidad Nacional de San Luis), subsidiado por Secretaría de Ciencia y Técnica Universidad Nacional de San Luis; Agencia Nacional de Promoción Científica y Tecnológica, y Proyectos de Investigación Plurianuales del CONICET. 2- Proyecto: A Psicologia nas Forças Armadas, na Igreja Católica e nas Cátedras. Bolsa de Produtividade e Edital Universal do CNPq, Cientista do Nosso Estado da Faperj, e Procientista da UERJ.

** Consejo Nacional de Investigaciones Científicas y Técnicas (CONICET)/Universidad Nacional de San Luis (UNSL), Argentina. Ejército de los Andes 950. Edificio Plácido Horas (4to Bloque), 2º piso. Box 71. CP 5700. San Luis. Argentina. - Teléfono: 54-266-4424027-Int. 4120. E-mail: mapineda@ unsl.edu.ar

*** Universidade do Estado do Rio de Janeiro, Conselho Nacional de Desenvolvimento Científico e Tecnológico (CNPq). Rua são Francisco Xavier, 524, bloco F, sala 10120 - CEP20559-900, Maracanã- Rio de Janeiro, RJ. Telefax: 55-21- 23340830. E-mail:amjaco@uol.com.br

\section{RES UMEN}

Se realiza un estudio historiográfico comparativo del desarrollo de la ciencia psicológica en Argentina y Brasil entre 1930 y 1980, analizando el papel de la psicología en la planificación estatal y las relaciones entre el modelo científico y el modelo profesional, basado en el análisis de variedad de fuentes primarias y secundarias. Hasta el inicio del proceso de creación de las carreras de psicología, se descubren procesos análogos de recepción y constitución del campo disciplinar e institucional, destacándose el lugar de la ciencia psicológica en la planificación estatal. Posteriormente, se registran las diferencias más significativas. En Argentina la cultura científica tendió a desplazarse afuera de dichas carreras, al igual que el apoyo estatal a la investigación psicológica. En Brasil, desde la década de 1960, el desarrollo tecnológico del sudeste y la dictadura militar propiciaron la difusión de una cultura psicológica en la clase media urbana. El Estado financió fuertemente los programas de posgrado y de investigación en las universidades.

Palabras clave

Argentina; Brasil; carreras; ciencia; profesión; psicología

\section{A B S T R A C T}

A comparative study of the development of the psychological science in Argentina and Brazil in the period 1930 and 1980 is carried out, based on the analysis of a variety of primary and secondary sources from a historical stand point. It is analyzed the role Psychology had in State planning and the relationships between scientific and professional model. Analogue processes of reception and constitution of the field and institutions are described until the creation of the psychology programs, when the greater State support and planning of Psychology development took place. Afterwards, the most meaningful differences are found between these countries. In Argentina scientific culture tended to be displaced from psychology programs, as well as the State support to psychology research. In Brazil, since the 1960 decade, the technological development in the southeast region, and the dictatorial government fostered the spread of a psychological culture among urban middle class people. The State strongly supported psychology postgraduate programs and research activity.

Keywords

Argentina; Brazil; programs; science; profession; psychology 
Como en gran parte del mundo occidental, en Argentina y Brasil la psicología como ciencia existe antes que la psicología como profesión. Con medio siglo de desarrollo en Sudamérica, las carreras de psicología han sufrido diversas transformaciones que han reflejado los cambios sociopolíticos y las culturas psicológicas de la región. En general, las carreras fueron delineadas bajo el modelo BoulderBogotá, que contemplaba una formación tanto científica como profesional (Baker \& Benjamin, 2000; Petersen, 2007; Gallegos, 2010). Sin embargo, las condiciones culturales, políticas y económicas que vieron nacer a las carreras de psicología sudamericanas auguraban un desarrollo científico muy modesto (Hereford, 1966).

Por tanto, cabe interrogarse, tras el proceso de definición social y regulación del rol profesional del psicólogo seguido desde la organización de las carreras de psicología, qué rumbo siguió la ciencia psicológica, la capacidad que han adquirido los psicólogos para contribuir científicamente a las nuevas demandas sociales y el espacio que ocupa la ciencia psicológica en la sociedad y en las políticas de Estado de las economías emergentes de Sudamérica. En este sentido, a cuarenta años de la Conferencia Latinoamericana para el entrenamiento profesional en psicología (Gallegos, 2010), asistimos a vivos debates sobre la formación universitaria del psicólogo en el contexto de evaluación y acreditación de carreras de psicología (Alzate-Medina, 2008; Baptista, 2010; Calabresi \& Polanco, 2011; Castro, 2004; Di Doménico \& Vilanova, 2009; Jacó-Vilela,1996; Mancebo, 1997; Sierra \& Bermúdez, 2005; Talak \& Courel, 2001; Vilanova, 2000; Villegas, Marassi, \& Toro, 2003). Desde la historia, se puede contribuir a su esclarecimiento, si analizamos críticamente el desarrollo científico y los espacios sociales conquistados por las comunidades de psicólogos. En ese sentido, si comparamos el estado de la ciencia psicológica en el período anterior a la creación de las carreras de psicología con el logrado posteriormente, vislumbraremos el panorama de las culturas científicas y profesionales gestadas en cada período, y las tensiones y resoluciones entre ellas. Así, contribuiremos a la comprensión de las matrices en la que se formaron los psicólogos.
Desde este punto de vista, nos interesa comparar estos procesos en dos países sudamericanos: Argentina y Brasil. Limítrofes entre sí, comparten características regionales, a la vez que presentan grandes diferencias culturales que nos permiten contrastar procesos y resultados. En ambos, el proceso de creación de las carreras de psicología se inició ya entrada la década de 1950. En los dos países, esta fue antecedida por un período de prometedora investigación psicológica básica y aplicada, que daba cuenta de un campo científico consolidado.

En efecto, al internarnos en las incipientes culturas psicológicas científicas de fines de siglo XIX y principios del XX de ambos países, se hace insoslayable la atención a los primeros laboratorios de psicología que, aún desde la compleja pluralidad de problemas y modelos que han visto nacer a la psicología como ciencia moderna, siempre han simbolizado la llegada de la ciencia positivista.

Tanto en Argentina como en Brasil, los primeros laboratorios de psicología experimental se fueron organizando alrededor del inicio del vigésimo siglo. En Argentina, generalmente se asociaban a las noveles cátedras con fines didácticos (Klappenbach, 1996). En Brasil, se vincularon la preparación de los profesores para la enseñanza primaria y la institución hospitalaria (Antunes, 1999; Centofanti, 2006). Pronto las prácticas psicológicas fueron ganando un lugar en la organización científica y laica de estos Estados modernos bajo los ideales del positivismo, procurando asesorar en temas de salud, justicia y educación, o dar respuesta a problemas sociales como el control de las masas en el contexto de las inmigraciones, la locura, la minoridad abandonada, etc. (Boarini, 2012; Carrilho, 1925; Falcone, 2012; Klappenbach \& Pavesi, 1994; Moreira, 1925). Ora montados con aparatos importados de Francia o de Alemania (Centofanti, 1982; Rossi, Ibarra, \& Kirsch, 2010), desde la década de 1920 en Argentina y en Brasil los laboratorios daban suficientes muestras de haberse convertido en productores de ciencia básica (Ardila, 1986; Papini, 1976; Radecki, 1929) y aplicada (Papini, 1978; Radecki \& Radecka, 1925). Paulatinamente, se recepcionaron las nuevas tecnologías de evaluación y medición basadas en procedimientos estadísticos, o test mentales, otro 
símbolo de rigor científico. No sin diálogo -a veces armónico y otras veces conflictivo-, con enfoques humanistas de raigambre filosófica, en esos vecinos países estas prácticas se extendieron masivamente -en Brasil desde la década de 1930, y en Argentina desde la de 1940-, asociadas a la psicotecnia y orientación profesional (Klappenbach, 2005; Rosas, 1997). Esto aconteció gracias a un proceso de expansión industrial y producción tecnológica estatal, en la que la psicología desplegaba su potencial, fundamentalmente en el campo educativo y laboral. Desde estas áreas, fue ganando el reconocimiento social y apoyo estatal que le abrieron el camino a la formación universitaria profesional (Cabral, 2004; Jacó-Vilela, 2002, Jacó-Vilela et. al, 2007; Klappenbach, 2006).

Desde este punto de vista, en el presente trabajo nos proponemos analizar el desarrollo de la ciencia psicológica en Argentina y Brasil desde 1930 hasta 1980. Particularmente, nos interesa analizar el lugar que ha ocupado la psicología en la planificación estatal y registrar los cambios, tensiones y asimilaciones entre el modelo científico y el modelo profesional, a partir de la creación de la carrera de psicología. Para ello, en primer lugar, trazaremos una descripción general de los centros que realizaban investigación básica o aplicada, dictaban cursos u ofrecían servicios desde la década de 1930 hasta la creación de las primeras carreras de psicología. En segundo lugar, describiremos el desarrollo de la investigación psicológica y su vinculación con estas hasta principios de 1980.

\section{Método y materiales}

Si bien no existen trabajos previos que comparen la psicología argentina y brasileña desde el enfoque propuesto, se han acumulado los datos necesarios para efectuar estas comparaciones. En efecto, nos preceden abundantes estudios que, desde diversas perspectivas, han abordado la figura de pioneros (p. ej., Campos, 2001a; Carpintero, 2006; Centofanti, 1982; Klappenbach, 2002b; Rosas, 1997; Rossi, 1997), han descripto instituciones y prácticas (p. ej., Ardila, 1979; Cirino, Miranda, \& de Souza, 2012; Dagfal, 2009; Ferrero \& Altamirano, 2011; Gentile,
2003; Herschmann \& Pereira, 1994; Jacó-Vilela, 2011; Jacó-Vilela et al., 2007; Klappenbach, 2002a; 2012; Lourenço Filho, 2002; Mustaca, 2006; Papini, 1976, 1978; Papini \& Mustaca, 1979; Piñeda, 2010; Rossi et al., 2001; Rossi et al., 2010; Scherman, 2009), han analizado la incidencia de publicaciones de psicología (p. ej., Klappenbach, 2001, 2005, 2009; Piñeda, 2005, 2012), han proporcionado una mirada global de la psicología de cada país (p. ej., Cabral, 2004; Klappenbach, 2006) o de América Latina (p. ej., Ardila, 1986; Klappenbach \& Pavesi, 1994). Además, se han recopilado y puesto en valor documentos y objetos que testimonian el desarrollo de la psicología como ciencia y profesión en estas latitudes (en Argentina: Archivo y Museo de Psicología Experimental Horacio Piñero de la Universidad de Buenos Aires, Archivo y Museo de Psicología de la Universidad Nacional de Córdoba, Archivo Plácido Horas y Museo de Historia de la Psicología de la Universidad Nacional de San Luis y en Brasil: Núcleo Clio-Psyché de la Universidade do Estado do Rio de Janeiro, Sala Helena Antipoff de la Universidade Federal de Minas Gerais, Museu de Historia da Psicologia de la Universidade Federal do Rio Grande do Sul). Por eso, desde el punto de vista metodológico, nos basaremos en variedad de fuentes primarias (informes y memorias, autobiografías, legajos, reglamentaciones universitarias, manuales y textos de psicología de incidencia en estos países, publicaciones de docentes e investigadores en psicología, inventarios de institutos y laboratorios, planes de estudio, programas de cursos de psicología) y testimonios de informadores clave que han sido relevados por nosotros en el curso de nuestras investigaciones sobre la temática y fuentes secundarias (especialmente informes sobre la investigación y las carreras de psicología).

\section{Resultados}

\section{Ciencia psicológica y profesionalización en Argentina}

Seis décadas antes de que en la universidad comenzaran a graduarse los primeros psicólogos, la ciencia psicológica estaba en manos de médicos, filósofos, 
educadores, abogados o clérigos que, para la década de 1930, contaban con ricos aportes a un campo científico consolidado (Klappenbach, 2006). La psicología de principios de siglo XX, como ciencia positivista había contribuido con la reforma de orden público del país y había recibido fuerte apoyo estatal (Vezzetti, 1981, Vilanova, 2001). Desde la temprana organización de los primeros laboratorios de psicología experimental entre 1891 y 1905 por parte de Víctor Mercante y Horacio Piñero, se habían sentado las bases del estudio científico de las funciones mentales normales y desagregadas y de las aptitudes sensoriales y motoras, combinando procedimientos psicofísicos con aparatos de laboratorio y test mentales que se siguieron desarrollando por algunas décadas en la Universidad Nacional de La Plata y la de Buenos Aires (Falcone, 2012; Klappenbach, 1996, 2006; Pantano, 1997; Papini, 1976; Talak, 2000, Vezzetti, 1988). Hacia 1920, con las recepciones de psicologías totalistas y la vuelta en vigor de planteos filosóficos sobre la persona humana y la libertad (Klappenbach, 1999), la anterior matriz positivista que buscaba superarse, más bien se vio completada y siguió produciendo frutos. Se destacaron investigadores como Christfried Jakob en psicofisiología cerebral (Triarhou \& Del Cerro, 2006), Enrique Mouchet, en lenguaje y pensamiento, percepción táctil en ciegos y emoción (Detrixhe \& Papini, 1978), Juan Cuatrecasas, en psicofísica de los sentidos, especialmente de la percepción óptica (Carpintero, Ferrándiz, \& Lafuente, 1997; Kurowski, 2001), o Alfredo Palacios, Carlos Jesinghaus y Gregorio Fingermann en psicofisiología aplicada a la orientación profesional, con los primeros estudios sobre la fatiga y accidentes de trabajo (Klappenbach, 2005; Rossi et al., 2010).

Pero la psicología aplicada al trabajo alcanzó su mayor difusión en la década de 1940. Bajo el gobierno del Presidente Juan Domingo Perón (19451955), se asistió a una política de sustitución de importaciones y expansión de la industria nacional. La orientación profesional se volvió una necesidad estatal, para asegurar la mejor mano de obra posible en cada puesto de trabajo (Klappenbach, 2005). Complementariamente, se impulsaba la orientación vocacional y se presentaba la psicología como el fundamento científico de la Escuela Nueva (Horas, 1949).

En ese contexto, se difundieron las bondades de los test mentales y florecieron alrededor de 35 institutos de psicología aplicada al trabajo, la educación, la medicina, las fuerzas armadas y la antropología, en su mayoría financiados por el Estado (Villaverde, 1954). Por ello, entre 1950 y 1953, se organizó la formación de especialistas en las universidades nacionales de Tucumán (carrera de Psicotécnico y Orientador Profesional, a cargo de Benjamín Aybar); Cuyo, Sede San Luis (Especialización en Psicología, dirigida por Plácido Horas) y Litoral, Sede Rosario (carrera de Asistente en Psicotécnica dirigida por Erminda Benítez de Lambruschini) (Gentile, 2003; Klappenbach, 2002, 2005; Rossi, 1997).

Así, desde la década de 1940, los enfoques psicofisiológicos de laboratorio eran reemplazados por la nueva tecnología: los test mentales, casi siempre derivados de la psicología francesa o española. La editorial Kapelusz editó dos de los íconos de la nueva cultura psicológica argentina: en 1947, el Manual de Orientación Profesional de Mira y López -quien se había radicado brevemente en Argentina-, y entre 1952 y 1961, los siete tomos del Tratado de Psicología Aplicada de Piéron (Klappenbach, 2005).

En este marco, es relevante la figura de Horacio Rimoldi. Él fue discípulo de Bernardo Houssay (Premio Nobel en Medicina de 1947) y con él se inició en psicología experimental en el Instituto de Fisiología. Con estudios doctorales desde 1939 en Oxford, Rimoldi rindió su tesis: "Ritmo y fatiga", publicada en 1946. Por acuerdo entre Houssay y la Universidad Nacional de Cuyo, ocupó allí la cátedra de Psicología Experimental y fundó el Instituto de Psicología Experimental. Formó un grupo de investigación en psicometría de las aptitudes y la inteligencia, entre quienes se contó Nuria Cortada, que había sido presentada a Rimoldi por Mira y López. Pese a que los trabajos de Rimoldi apuntaban a temas valorizados por la planificación estatal posterior a 1945, con la llegada de Perón al poder y su intervención en las universidades nacionales, Rimoldi se trasladó a Chicago en 1949, para continuar su formación en psicología matemática junto a Louis Thurstone. En la Universidad de Loyola, Rimoldi 
fundó un laboratorio psicométrico donde permaneció hasta 1970, investigando sobre inteligencia y resolución de problemas y formando y asesorando investigadores de diversos países (Carpintero, 2006; Rimoldi, 1995).

Pero el complejo escenario argentino de la década de 1940 no ha sido uniforme en torno a la psicotecnia. Prueba de ello ha sido la organización de la Asociación Psicoanalítica Argentina en 1942 (Balán, 1991; Plotkin, 2003; Vezzetti, 1989), desde la que los psicoanalistas se fueron convirtiendo en nuevos agentes sociales. Su protagonismo se fue extendiendo más allá de la clínica, desde su entrada al plantel docente y directivo de varias carreras de psicología durante la década de 1960 (Ardila, 1979; Saforcada, 1969) y la difusión del psicoanálisis en la cultura. Este modelo entraría en franca tensión con la psicotecnia fundacional de las carreras.

En efecto, el auge de la psicotecnia y la orientación profesional en el marco de peronismo fue generando las condiciones para que en el Primer Congreso Argentino de Psicología celebrado en Tucumán en 1954, se declarara la necesidad de organizar la formación universitaria de psicólogos. Así, aún con las contradicciones políticas generadas tras la caída de Perón, entre 1954 y 1964 se fundaron 14 carreras de psicología: 6 en universidades nacionales, 2 en instituciones provinciales y 6 en universidades privadas (Gentile, 2003; Klappenbach, 2003). Los primeros planes de estudio tendían a contemplar la formación científica y profesional (Horas, 1961), y los Departamentos e Institutos de universidades nacionales producían investigación. Estos han reflejado las tensiones entre la ciencia psicológica y el modelo profesional.

En la Facultad de Filosofía y Letras de la UBA, Enrique Mouchet había organizado en 1930 el prestigioso Instituto de Psicología. Sin embargo, el mismo declinó su actividad tras la creación de la carrera de psicología, careciendo de investigaciones hacia 1970 (Papini \& Mustaca, 1979). En 1958, se creó el Departamento de Psicología, por un año a cargo de Marcos Victoria, sucedido hasta 1960 por Enrique Butelman. Este fue socio de Jaime Bernstein en la Editorial Paidós, especializada en obras de psicología y educación, con tendencia a la difusión de una psicología de corte clínico (Klappenbach, 2001). Un año después, Butelman fue sucedido por Manuel Solari, y entre 1961 y 1962 por Telma Reca. Desde 1957, Reca dirigió el Centro de Psicología y Psicopatología de la Edad Evolutiva dependiente del Rectorado, vinculando las Facultades de Filosofía y de Medicina. También en el Rectorado, en 1957 se creó el Departamento de Orientación Vocacional a cargo de Bernstein y luego de Nuria Cortada (Rossi et al., 2001).

Más allá de las transiciones a nuevos modelos en los inicios de la carrera, la producción científica se vio resentida tras el golpe de Estado de 1966. Este provocó la intervención de las universidades nacionales, masivas renuncias de los docentes de la UBA y el desmantelamiento de la Editorial Universitaria (Eudeba) que había sido prolífera incluso en textos de psicología (Rossi et al., 2001). Para 1968, comprobamos floreciente actividad investigativa fuera de la carrera de psicología. En la Facultad de Medicina, el equipo dirigido por Jorge Insúa en la cátedra de Psicología Médica (psicofisiología y psicoterapia) y el de Jorge Affani en Fisiología Animal (mecanismos nerviosos del sueño, aprendizaje y memoria). Desde mediados de 1970, se sumaron el grupo de Juan Izquierdo en la Facultad de Farmacia y Bioquímica (psicofarmacología y condicionamiento simple y operante) y la cátedra de Fisiología Animal de la Facultad de Ciencias Exactas y Naturales a cargo de Mauricio Papini, integrada entre otros por Alba Mustaca (aprendizaje y memoria). Este equipo luego nutrió los planteles del Instituto de Biología y Medicina Experimental (IByME) -fundado por Houssay en 1944, asociado a la Facultad de Medicina de la UBA desde 1977-, y del Laboratorio de Psicología Experimental (PSEA) del Instituto de Investigaciones Médicas Alfredo Lanari (IDIM-CONICET) fundado en 1957 (Mustaca, 2006; Papini \& Mustaca, 1979). Por otra parte, en 1968 se creó en la Facultad de Ingeniería el Laboratorio de Investigaciones Sensoriales (LIS), que en 1972 pasó a la Facultad de Ciencias Médicas y se incorporó a CONICET. El LIS fue organizado por Miguelina Guirao, graduada en Filosofía de la UBA en 1957. Fue miembro del Departamento de Orientación Vocacional dirigido por Cortada. En 
1960, dictó los cursos de Orientación Profesional, Psicología Aplicada y Psicología Experimental en la carrera de psicología de Rosario (Programas de los cursos citados). Inició sus estudios doctorales sobre procesos cognitivos en el Instituto de Psicología de la Universidad Católica de Milán. La dirigió Agostino Gemelli, neurólogo, psicólogo experimental, especialista en psicometría y orientación profesional y fraile franciscano de gran incidencia en Argentina cuando se celebró el Primer Congreso Argentino de Psicología (Gemelli, 1954) y aún en los años sesenta (Piñeda, 2005). Ya en Argentina, Guirao se entrevistó con Houssay, Presidente de CONICET, quien le recomendó completar sus estudios desde la perspectiva sensorial con Michael Stevens en Estados Unidos. CONICET la becó y a su regreso la incorporó como investigadora (Guirao, 2008).

En la Universidad Nacional de La Plata, el Instituto de Psicología de la Facultad de Humanidades y Ciencias de la Educación estuvo dirigido por Luis María Ravagnan, en los inicios de la carrera de psicología, y entre 1965 y 1972 por Mauricio Knobel (Klappenbach, 2009). Durante el segundo período, se registró la mayor cantidad de publicaciones de los docentes que lo integraban (p. ej., Knobel, Nuria Cortada, Edgardo Rolla, Juan Cuatrecasas, Ravagnan y Ricardo Musso), mayormente en psicología clínica y psicología evolutiva. El Instituto también promovió servicios de evaluación de la personalidad, asesoramiento psicológico y cursos y conferencias. Tras su cierre en 1972, el Departamento de Psicología a cargo de Celia Paladino producía escasa investigación (Piñeda, 2012). Con el golpe militar de 1976, identificándose la carrera de psicología con la penetración ideológica subversiva, se interrumpió su inscripción en la UNLP. El Departamento fue cerrado en 1979 y la Revista de Psicología, editada desde 1964, fue discontinuada (Klappenbach, 2009). Por otra parte, desde 1959 en la cátedra de Psicología Médica de la Facultad de Medicina se investigaba en psicopatología y psicofarmacología (Rubén Córsico y Neli Pastoriza) (Papini \& Mustaca, 1979).

En la Facultad de Filosofía y Humanidades de la Universidad Nacional de Córdoba (UNC), Raúl Alberto Piérola organizó el Instituto de Psicología en 1956, y como quería orientar el Instituto hacia las crecientes demandas industriales de Córdoba, buscó el apoyo de especialistas y del Consejo $\mathrm{Na}$ cional de Investigaciones Científicas y Técnicas (CONICET) que organizó Houssay en 1958, sin lograr su cometido (Ferrero \& Altamirano, 2011).

Entre los asesores convocados en 1957, estuvo Rimoldi que se encontraba en Argentina porque lo habían designado Profesor Titular de Psicología II en la recientemente creada carrera de psicología de la UBA, cargo al que renunció sin ejercer. En la UNC, fue designado Profesor Extraordinario y dictó: Psico-estadística y teoría de las pruebas mentales, Metodología y Psicotecnia y Orientación Profesional (Rimoldi, 1995; Sherman, 2009). Pero, tampoco renovó su contrato con la UNC. Retomó su trabajo en Chicago hasta 1970, cuando regresó al país porque CONICET le confirió el máximo rango como investigador, y fue nombrado Profesor Titular en la Facultad de Ciencias Exactas y Naturales de la UBA. Al año siguiente, organizó el Centro Interdisciplinario de Investigaciones en Psicología Matemática y Experimental (CIIPME), al que también se incorporó Nuria Cortada (Rimoldi, 1995). En esa época, retomó el contacto con la UNC, asesorando investigadores que se establecieron en el Centro de Investigaciones Acústicas y Luminotécnicas (CIAL) y a su discípula Hermelinda Fogliatto, quien se había formado con él en el Laboratorio de Chicago en temas relativos al entrenamiento en la resolución de problemas (Rimoldi, 1995). Al llegar a la UNC, al menos entre 1967 y 1973, Fogliatto ocupó diversas cátedras (Orientación Profesional, Método y Técnicas de Investigación Psicológica, Análisis Factorial) y condujo investigaciones. Ingresó como investigadora del CONICET en 1975 (Legajo en Archivo de CONICET).

El CIAL había sido organizado en 1952 por el Ingeniero Guillermo Luis Fuchs en la Facultad de Arquitectura y Urbanismo (Ferrero, Iemme Khoury, \& Todisco, 2013). Interesado por ampliar los estudios interdisciplinarios, convocó a alumnos de la carrera de psicología para realizar sus tesis en temas de sonoridad, luminancia y percepción espacial. Las dirigió Fuchs y las codirigieron Fogliatto y el italiano Franco Murat, exdocente de la carrera de 
San Luis. En 1963, la primera tesis fue de Enrique Saforcada, posteriormente docente de la carrera hasta el golpe de Estado de 1976. Entre las logradas en los cinco años siguientes, estuvieron las de Cristina Biassoni y Ana María Verzini (Listado de tesis en Archivos CIAL/CINTRA), luego becarias e investigadoras de CONICET en el CIAL (comunicación personal con Biassoni y Verzini, 6/11/13).

Juan Seguí también se contó entre los primeros investigadores de la carrera de psicología de la UNC, en el campo del lenguaje, antes de partir a Francia, en 1965. Allí fue becario de Paul Fraisse, Director del Laboratorio de Psicología Experimental de la Sorbona, puesto en que Seguí fue sucesor (Muñoz \& Mañas, 2012).

En la Universidad Nacional de Cuyo, Plácido Horas organizó el Instituto de Investigaciones Psicopedagógicas, en 1956. Desde la creación de la carrera de psicología y hasta el cierre del instituto en 1972, allíse coordinó la formación, investigación y servicios en psicología. El Instituto se convirtió en un centro académico de psicología comportamental, abierto a influencias europeas, latinoamericanas y norteamericanas, contrastante con las tendencias mayoritarias de las demás carreras de psicología perfiladas hacia el psicoanálisis y la clínica (Piñeda, 2010). Este grupo se fue consolidando gracias a la obtención de diversos recursos, no obstante limitados y discontinuados. Así, durante la década de 1960 se pudieron conseguir subsidios del CONICET para becas de formación de posgrado en Inglaterra: la de Eva Mikusinski entre 1964 y 1966 con Hans Eysenck, en evaluación de la personalidad, y la de Claribel Barbenza entre 1966 y 1968 con William Tempest, en psicoacústica.

Asimismo, el CONICET subsidió investigaciones aplicadas (Mikusinski, Horas, Gialluca, 1969) y hacia 1980 incorporó a Mikusinski a la carrera de investigadora y otorgó algunas becas bajo su dirección. Por otra parte, la Organización de Estados Americanos subsidió la compra de equipamientos de laboratorio (Piñeda, 2010), y la Sociedad Interamericana de Psicología financió viajes de estudios de su vicepresidente, Plácido Horas, a laboratorios europeos y norteamericanos. Después de 1973, cuando la carrera de psicología pasó a la flaman- te Universidad Nacional de San Luis (UNSL), se contrataron profesores visitantes como Juan Seguí, Víctor Rojas, Rubén Ardila y Antonio Battro.

En 1976, se creó la Secretaría de Ciencia y Técnica de la UNSL, acreditándose los proyectos de investigación sobre evaluación de la personalidad desde la perspectiva de Eysenck (Mikusinski); funciones de sonoridad (Barbenza); psicofármacos y condicionamiento (Emilio Alaggia); orientación vocacional (Elena Ossola) y criminología (Horas). Con su trabajo sostenido, en 1980 se organizó del Laboratorio de Psicología Experimental a cargo de Barbenza. El débil apoyo institucional lo sostuvo solo dos años. Sin embargo, esta comunidad de investigadores, en 1987 propició la primera sede de la Asociación Argentina de Ciencias del Comportamiento -que hoy es la organización nacional miembro de la Unión Internacional de Psicología Científica (IUPsyS)-, siendo Barbenza su primera Presidente (Piñeda, 2010).

Así, hemos descripto un proceso de vaciamiento de las carreras de psicología de su capacidad científica por diversos factores. Primero, ideológico, con un clima anti-experimentalista (Ardila, 1979; Papini, 1978) potenciado por el avance del psicoanálisis y una cultura humanista de tinte nacionalista y latinoamericanista que vinculaba los modelos experimentales a los intereses norteamericanos. La psicotecnia y psicología experimental mantuvieron alguna presencia, pero fueron perdiendo vigencia, desapareciendo del currículo (Horas, 1961, 1981). La investigación no era considerada una opción laboral rentable (Horas, 1981). Consecuentemente, se fueron desplazando docentes de perfil científico a otras facultades o al exterior.

Esto también se debió a factores político-económicos. El apoyo presupuestario se diluyó durante la década de 1960, con los agravantes de los golpes de 1966 y 1976. Con este último hubo una política general de desplazamiento de recursos económicos para la investigación de las universidades a institutos externos, pequeños y políticamente controlables (Bekerman, 2009) (p. ej., Instituto Rosario de Investigaciones en Ciencias de la Educación, organizado en 1977 por Ricardo Bruera en convenio entre CONICET y la Universidad Nacional de 
Rosario), para contrarrestar la penetración ideológica subversiva, uno de cuyos focos se consideraba la carrera de psicología. Además, la formación del psicólogo era evaluada deficiente en el marco de confrontaciones con el campo médico por la delimitación del rol profesional clínico (Klappenbach, 2012). Esta visión alcanzaba esferas decisivas con el médico Fermín García Marcos, Secretario de Ciencia y Tecnología de la Nación en 1978 y Presidente de CONICET en 1979, que había elevado un lapidario informe (Barrionuevo \& García Marcos, 1975) cuando era Secretario de Planeamiento del Consejo de Rectores de Universidades Nacionales (Klappenbach, 2009).

\section{Ciencia psicológica y profesionalización en Brasil}

Gracias al detallado trabajo de Marina Massimi $(1990,2006)$ sobre la obra jesuítica del período colonial, conocemos las ideas psicológicas de orientación tomista que guiaron el discurso jesuita sobre el indígena brasileño. Estas constituyeron las construcciones teóricas para comprender el nuevo ser encontrado en las lejanas tierras allende el mar, que finalizaron con las dudas sobre la existencia del alma en los indígenas: dotados de inteligencia, voluntad y memoria según la conceptualización tomista de las facultades del alma (Nóbrega, 2006). En esos momentos, en que Iglesia y Corona, cruz y espada no eran instituciones independientes, la relación de la disciplina psicológica y el Estado era estrecha. Estas ideas entraron en crisis en el siglo XIX con el "advenimiento de las ideas nuevas" europeas (Romero, 1926): materialismo, evolucionismo, positivismo, Wundt, Ribot, Janet, que asimilaron los intelectuales imbuidos del liberalismo, comprometidos con el proceso de abolición de la esclavitud (1888) y la proclamación de la República (1889). Bajo el nuevo modelo de Estado y sociedad regidos por los principios liberales de igualdad, médicos y abogados se apropiaron de la psicología científica.

El proyecto republicano era civilizatorio, pretendía transformar un país rural, analfabeto e insalubre en una nación moderna según modelos europeos. El sanitarismo (Herschmann \& Pereira, 1994) y la educación de los ciudadanos (Gondra, 2004) eran consideradas grandes herramientas para subsidiar el proyecto, y la psicología se financiaba en dicho contexto. En ese sentido, el primer laboratorio de psicología experimental habría sido construido en el Hospicio Nacional de Alienados en 1897 (Anita Cabral, 2004) y la primera tesis de psicología experimental del médico Henrique Roxo, "Duración psíquica de los actos elementales en alienados", fue defendida en 1900 en la Facultad de Medicina de Rio de Janeiro vinculada al Hospicio. Por otro lado, la psicología crecía gracias a la reforma educacional (Decreto 981/1890) que abrió paso a la introducción de la psicología en la enseñanza por considerarse la base de la misma (Lourenço Filho, 2002). Por eso, se organizaron laboratorios experimentales en las escuelas normales que formaban maestros. El primero fue el organizado en el museo Pedagogicum perteneciente al Ministerio de Instrucción Pública, Correos y Telégrafos a cargo de Manoel Bomfim, con equipamientos venidos de París y la ayuda de Alfred Binet. Otros laboratorios fueron creados en las escuelas normales públicas de las principales ciudades como São Paulo (por Clemente Quaglio y Lourenço Filho) y Belo Horizonte (por Helena Antipoff) (Antunes, 1999; Centofanti, 2006).

En la década del veinte, de la misma forma que en Argentina, en Brasil los aparatos experimentales comenzaron a sustituirse por los test psicológicos. Aunque a diferencia de su vecino país, en Brasil los aparatos no desaparecieron por completo, sino que siguieron conviviendo con los test mentales. Pero la transición registrada fue criticada por quienes sostenían que los psicotécnicos se hacían pasar por científicos, de la misma forma que los curanderos por sabios (Radecki, 1929). Aún así, los test ayudaron a la rápida difusión y autonomización de la ciencia psicológica, al mismo tiempo que esta revelaba su utilidad social (Rose, 2008). En el contexto de educación laica, gratuita y obligatoria para todos, el movimiento de la Escuela Nueva consideraba los test como un instrumento para la construcción del dispositivo de las clases homogéneas. La vigencia de estos se mantuvo durante el primer gobierno de Vargas con figuras como Anísio Teixeira y Lourenço Filho, y aún tras el alejamiento de Teixeira 
cuando el gobierno se transformó en dictadura. En diversas investigaciones se advierte el patrocinio de la Liga Brasileña de Higiene Mental (Frossard, 1930; Leme Lopes, 1930, 1929; Lopes, 1931) como de los diversos órganos estatales que comenzaban a utilizar los test (Antipoff, 1930; Pernambucano, 1930). También se crearon test brasileños, como el de inteligencia para analfabetos "ABC" de Lourenço Filho (2008), sin duda el más famoso, con traducciones en castellano por Kapelusz, así como al inglés y francés.

Entre 1910 y 1930 la formación en psicología era autodidáctica, mediante libros importados, viajes de corta duración al exterior e invitación de figuras extranjeras de marcada francofilia a Río de Janeiro, São Paulo y Belo Horizonte, algunas con financiamiento estatal: George Dumas (1866-1946) en 1912 y otras fechas, Henri Pierón (1881-1964) en 1921 y 1927, Wolfgang Köhler (1887-1941) en 1930; Edouard Claparède (1873-1940) y Théodore Simon (1873-1961), León Walter. Entre 1937 y 1940, ofrecieron conferencias en Río de Janeiro nombres como Pierre Janet (1859-1947), Henri Wallon (1879-1962) y Rudolph Dreikurs (1897-1972) (Anita Cabral, 2004).

A las cátedras existentes desde la década de 1910 en las Escuelas Normales, a partir de los años de 1930 se le sumaron las primeras cátedras en instituciones universitarias de nivel superior, cuando otros europeos visitaron el país. Generalmente llegaban a la Universidad de Brasil (UB), Río de Janeiro, y a la Universidad de São Paulo (USP), que eran las dos principales. A la Facultad Nacional de Filosofía de la antigua UB llegaron Etienne Sorineau (de 1934 a 1937) y André Ombredane (de 1940 a 1945). A la USP, Jean Maügue (de 1935 a 1944), Otto Klineberg (de 1945 a 1947) y Fred Keller (de 1961 a 1962). En 1964, Fred Keller también estuvo en la Universidad de Brasilia (UnB) (Cirino, Miranda y Cruz, 2012; Penna, 1992). Más allá de los efectos civilizadores que estos personajes causaron, fueron pocas las recepciones efectivas, pero estas generaron las principales líneas investigativas de la época. Claparède y la línea educacional ginebrina se reflejó principalmente en Belo Horizonte donde una de sus colaboradoras, Helena Antipoff (1892-
1974) se estableció en 1929 para dirigir el "Laboratório de Psicologia da Escola de Aperfeiçoamento de Professores", dentro del proyecto de reforma educacional del gobierno de la entonces Provincia de Minas Gerais (Campos, 2001b). Helena Antipoff organizó varias instituciones y fue catedrática de Psicología de la Educación de la Facultad de Minas Gerais. En sus investigaciones principalmente utilizó test, creando uno llamado "Mis Manos" (MM) (Antipoff, 1975). Su principal contribución, fue la creación de diversas instituciones para la niñez (Campos, 2001b). Otto Klineberg a su vez contribuyó con la maduración de una psicología social "brasileña" aún embrionaria en la USP, proyecto de Anita Cabral que realizó su Maestría en Estados Unidos con los gestálticos allí exiliados. Fred Keller y el análisis experimental del comportamiento, más tardío en nuestro análisis, tuvo una recepción que sobrepasó los espacios institucionales iniciales (USP y UnB) hacia el norte y sur del país, gracias al prestigio académico y a la capacidad de articulación de su principal asistente en la USP Carolina Bori (Tassara, 1998). En la UB, se enfatizó principalmente en los estudios fenomenológicos y gestálticos por influencia del catedrático Nilton Campos. Otro profesor de esta universidad, también catedrático de Psicología Social de la Universidad del Distrito Federal, fue un asiduo investigador sobre el negro brasileño a la luz del psicoanálisis (cf. Ramos, 2001). Su libro Introducción a la Psicología Social (2003) representa una amalgama de lo conocido en la época en dicho campo.

Mientras tanto, fuera de las cátedras, en instituciones dedicadas a la práctica profesional se realizaban investigaciones aplicadas, siendo la dicotomía entre ciencia básica y aplicada un paradigma dominante en la época. Entre 1930 y 1960, muchas instituciones se destacaron. Mantendremos nuestro foco de análisis en los mismos tres Estados del sudeste del país antes mencionados, porque en este período era rara la presencia de la práctica psicológica en otras regiones, dado que la difusión de las mismas por el mapa brasileño siguieron el mismo recorrido que el proceso de urbanización, industrialización y modernización tecnológica (Figueira, 1985; Russo, 1993). Una de las instituciones 
profesionales más relevantes ha sido el Instituto de Selección y Orientación Profesional (ISOP) de la Fundación Getúlio Vargas (FGV). Creada en 1944, preparaba personal de alto nivel para la administración pública y privada del país, acorde al nuevo perfil de país en el contexto de transformaciones y crecimiento industrial. El ISOP "contribuía al proceso de modernización de la relación trabajadorcapital, con base en los principios de la psicotecnia y la psicología racional” (Motta, 2005, p. 127), estudiando el ajuste de las aptitudes del trabajador a los requisitos psicofisiológicos del trabajo. Realizaba selección y orientación de personal y formaba psicotécnicos al mismo tiempo que conducía investigaciones científicas. Mira y López (1886-1964) fue invitado a dirigir el ISOP. En 1947 se estableció en Río de Janeiro para dirigir el ISOP y permaneció allí hasta su muerte. El ISOP rápidamente se convirtió en un centro de divulgación de psicología, abriendo nuevas filiales en otros estados del país. En 1949 fundó la revista Arquivos Brasileiros de Psicotécnica, y luego sus técnicos constituyeron la Asociación Brasileña de Psicotécnica (ABP). Los trabajos publicados en dicha revista, de autores no restringidos al personal del ISOP, incluso extranjeros, la mitad de éstos mujeres (Jacó-Vilela et al., 2007), tenían un carácter eminentemente práctico, relatando investigaciones o enseñando cómo realizar diversas actividades como el análisis profesiográfico. Tras la muerte de Mira y López, el ISOP se mantuvo activo, aunque sufriendo cambios de orientación, enfatizando la investigación y desde 1971 ofreciendo un posgrado en psicología.

Otra institución relevante en la psicología de Río de Janeiro fue el Centro de Orientación Juvenil (COJ), creado por el Ministerio de Salud como órgano de la Sección de Orientación Social de la División de Protección Social del Departamento Nacional de Crianza (DNCr), respondiendo al ideal de educar a los niños para preparar al ciudadano de mañana y construir un país civilizado y saludable. El DNCr atendía la infancia pobre no peligrosa, y bajo la planificación de Helena Antipoff y Emilio Mira y López organizó el COJ en 1946, la primera clínica pública federal para orientación psicológica de los jóvenes, cuyo cuidado hasta entonces se restringía a la educación. Su equipo también contaba con Servicio Social y Psiquiatría. Inicialmente volcado al psicodiagnóstico y la orientación, posteriormente, el COJ también ofreció tratamiento psicoterapéutico. Por sus contactos con el ISOP, en los años cincuenta el personal del COJ se nutrió de los aportes de Mariana Alvin que trabajó con Carl Rogers en Estados Unidos.

Por su parte, en Minas Gerais, las principales instituciones del período fueron las organizadas por Helena Antipoff como la Sociedad Pestalozzi de Minas Gerais (1932) y la Hacienda del Rosario (1939) en torno de la cual se aglutinaron otras instituciones, como el Instituto Superior de Educación Rural (1952) que, posteriormente, se transformó en fundación con apoyo gubernamental. Todas estas instituciones se orientaban a los objetivos principales de Antipoff respecto de los niños "excepcionales" en sentido físico, mental o social, a la formación de profesionales para el magisterio y a la preocupación por el hombre de campo. En los años cincuenta también se creó el Departamento de Orientação e Treinamento do Banco da Lavou$r a$, de orden privado, y que inauguró prácticas no tradicionales para la entonces clásica psicología industrial, como las dinámicas de grupo (Goulart $\&$ Santos, 2011). Su director, Pierre Weil luego fue profesor de la Universidad Federal de Minas Gerais, invitado por uno de sus asistentes en el Banco que ya era docente en dicha universidad, Celio García (Bomfim, 2001).

En São Paulo, centro industrial y financiero del país, por otro lado, muchas fueron las instituciones y sus contribuciones. En el escenario educacional se destacan los Servicios Educacionales. Citamos aquí solo el Servicio de Psicología Aplicada (SPA) de la Secretaria de Educación y Salud Pública de São Paulo, por haber sido creado por Lourenço Filho en 1930 cuando era Director de Instrucción Pública. El SPA evaluaba alumnos (con el test ABC u otros), ofrecía pasantías a los alumnos de la Escuela de Profesores, realizaba estudios solicitados por las escuelas y servicios públicos y ofrecía cursos sobre psicotecnia para profesores. En 1938 el Gobierno del Estado decretó su pasaje a la Sección Educación de la Facultad de Filosofía, Ciencias y Letras de la 
Universidad de São Paulo, lo cual es considerado la base para la futura creación de la carrera de psicología en aquella institución (Antunes, 1999).

La Clínica de Orientación Infantil (COI), también vinculada a la Secretaria de Educación del Estado de São Paulo, fue creada en 1938 por Durval Marcondes, aún bajos los efectos del movimiento higienista pero con fuerte influencia del psicoanálisis. Marcondes propuso un nuevo servicio de higiene mental escolar que, por medio de la formación de profesoras, se enfocaba en el estudio del ambiente familiar con un equipo multidisciplinar. El énfasis higienista suponía la segregación del alumno excepcional en clases especiales (Lima, 2011).

Cabe resaltar que en este período se organizaron en São Paulo dos asociaciones relevantes en el proceso de reglamentación de las carreras de psicología: la Sociedad de Psicología de São Paulo (1945), cuyo Boletim de Psicologia, fundado en 1949 es uno de los más antiguos de Brasil; y la Asociación de Psicólogos de Brasil, actualmente Asociación Brasileña de Psicología (1954).

El anteproyecto de reglamentación de las carreras de psicología fue propuesto por la Asociación Brasileña de Psicotecnia y publicado en los Archivos Brasileños de Psicotecnia en 1954. Por su carácter dicotómico entre teoría y práctica y principalmente por el conflicto existente con Nilton Campos y Mira (Mancebo, 1997), fue llevado adelante principalmente por las asociaciones paulistas con Anita Cabral y Arrigo Angelini al frente (Baptista, 2010). El proyecto respondía a la realidad de que ya estaban funcionando diversas carreras de psicología, en instituciones privadas católicas, y por tanto más ágiles en su planeamiento administrativo: PUC-RIO (1953), PUC-RS (1954), Universidad Católica de Minas Gerais (1959) y Universidad Católica de Pernambucano (1962), siendo la Universidad de São Paulo (1957) la única institución pública que abrió dicha carrera antes de la reglamentación legal (véanse las entradas sobre esas instituciones en Jacó-Vilela, 2011). Con el trabajo conjunto de las tres asociaciones entonces existentes y fuerte apoyo de los profesionales que actuaban en este campoen su mayoría mujeres-, el proyecto se transformó en la Ley № 4.119/62. Reglamentando la profesión y las carreras, así como su currículo mínimo (Parecer 403/61 del Consejo Federal de Educación), la formación de los psicólogos preveía una carrera de cinco años de duración, como se mantiene hasta hoy. Se estableció bajo el modelo positivista con "separación entre teoría y aplicación, ciencia y práctica, definiendo el objeto de estudio: "la" personalidad, "el" desarrollo... y no menos revelador, "la" psicología social explicitando un área dedicada al individuo en relación, en el encuadre general del estudio "del" individuo (Jacó-Vilela, 1996, p. 34).

El espíritu eufórico de la época autorizaba también a las instituciones públicas a crear sus carreras, como en el caso de la Universidad de Brasil (hoy Universidad Federal de Río de Janeiro) y la Universidad del Estado de Guanabara (hoy Universidad del Estado de Río de Janeiro), que las organizaron en 1964. La carrera de grado en psicología habilitaba al profesional a actuar en diversas áreas (clínica privada, diferentes instituciones como la escuela, organizaciones de trabajo, justicia, instituciones públicas y privadas de salud, penitenciarías, asistencia a jóvenes en conflicto con la ley, etc.), el diploma de licenciado en psicología (obtenido por la acreditación de disciplinas pedagógicas sumadas al currículo de la carrera de psicología) habilitaba al magisterio en el nivel secundario.

Con todo, la dictadura militar se instauró en el país justamente en esta época, el 1 de abril de 1964, y duró 21 años, hasta 1985. Atravesó varios períodos: el de perseguir a los enemigos declarados del régimen (1964-1968), el de que todos son enemigos potenciales (1968-1974), el de una tentativa de ablandamiento (1974-1979) y un proyecto de apertura (1979-1985). La psicología "oficial" de Brasil como han destacado diversos autores como Coimbra (1995), se desarrolló bajo el signo del orden aceptando la opresión. Aunque también existe la impresión de que la mayoría de los psicólogos fue indiferente a los efectos que su actuación profesional podía tener en relación con el proyecto político de la dictadura. Parece haber habido psicólogos enmudecidos por el sistema, establecidos en el servicio público, con dificultades para conseguir otro trabajo, como también presos, exiliados, torturados y muertos. Pero aún no hay estudios precisos al 
respecto que permitan conclusiones fidedignas. Lo que sí podemos afirmar es que la dictadura ejerció sus efectos en la constitución de las subjetividades (Velho, 1978) y en la formación cultural de los universitarios. Había pocas editoriales dedicadas a este público en Brasil, y todas ellas sufrían presión de la dictadura. Por tanto, los estudiantes de psicología de los años iniciales solo tenían acceso a las obras de psicología publicadas por la Biblioteca de Educación de la Editorial Melhoramentos y a las obras traducidas por Dante Moreira Leite y Carolina Bori, que componían un curso introductorio de psicología publicado por la Editorial Herder. Fuera de esto, se accedía a las editoriales argentinas (principalmente Paidós y Kapelusz) (Jacó-Vilela, 2012). Por su parte, las instituciones católicas también produjeron sus propias lecturas de psicología como los libros de Theobaldo Miranda dos Santos (1956, $1958,1962,1963,1966)$ y, principalmente en las instituciones salesianas, traducciones de las obras de Gemelli (Ferraz, 2014.), cuya mezcla de tomismo con psicología experimental parecía reconciliar la teología con la modernidad. Hasta entonces, el mercado editorial interesado en la psicología, que estaba volcado a la educación, descubrió las carreras y comenzó a publicar libros básicos y manuales.

El crecimiento de las publicaciones en psicología y su adopción en la enseñanza ocurrió a mediados de los años ochenta. Atribuímos ese hecho a diversos factores. El posgrado que se había iniciado en 1964 en la UnB y luego había sido suspendido, se reafirmó a partir de 1967 con el curso de la PUC-RIO, cuando la dictadura militar invirtió fuertemente en este nivel de enseñanza. El creciente número de carreras de grado, a partir del incentivo estatal a la iniciativa privada para la creación de las mismas, Facultades y Universidades y la psicologización de la sociedad brasileña desde los años setenta (cf., DaMatta, 1985; Figueira, 1981; Russo, 1993) llevó a un interés cada vez mayor por la psicología. Por tanto, se produjo un proceso de reflexión sobre la necesidad de conocimiento sobre la realidad brasileña, y en consecuencia, crecieron las producciones y la enseñanza de la psicología basadas en ella.

Por otro lado, es importante resaltar que las cátedras perdieron su vigencia, ya que fueron abolidas del sistema educacional brasileño con la reforma universitaria al final de los años sesenta (Ley № 5.540, 28/11/86) (Saviani, 2008). Otra consecuencia de dicha reforma fue la introducción del Departamento, como elemento principal de la vida universitaria y del sistema de créditos. Dentro de su política de desarrollo tecnológico, el gobierno militar, mediante los órganos de financiamiento como el Consejo Nacional de Desarrollo Científico y Tecnológico (CNPq) y la Coordinación Nacional de Perfeccionamiento de Personal de Nivel Superior (CAPES), pasó a brindar mayor apoyo, inclusive financiero, al posgrado. Desde ese momento, este se divide en dos carreras: la de maestría, que ha tenido una duración variable, hoy de 24 meses, y la de doctorado, hoy con 48 meses. El objetivo de ambos es, inicialmente, formar profesores para el nivel universitario en vistas al aumento de carreras de nivel superior, principalmente en las instituciones privadas, con incentivo gubernamental. La relevancia de los títulos depende de la demanda social. Las carreras de posgrado se localizan desde los años de 1960 principalmente en las instituciones públicas y en las confesionales, y desde mediados de 1990 las agencias exigen resultados (producción de publicaciones) de investigación científica financiada por ellas. En este sentido, los cursos de grado aparecen como auxiliares en la medida en que proveen de alumnos que serán becarios de iniciación científica y comenzarán su entrenamiento en ciencia.

De ese modo, se ha dado un gran paso de disolución de la dicotomía entre teoría y práctica de los años cincuenta, al mismo tiempo que se ha producido una ruptura con el proyecto de psicología brasileña gestada entre las décadas de 1930 y 1940. En efecto, en los setenta, en varios lugares, tanto psicólogos académicos como profesionales comenzaron a sentir insatisfacción con su práctica restringida a enfoques norteamericanos. Sus planteos llevaron inicialmente a una ampliación de la práctica (los llamados proyectos comunitarios) (Gonçalves, 2013) y concluyeron en reflexiones académicas con el gran debate de los inicios de 1980: "iqué profesional queremos formar?" (JacóVilela, 1996). 
Esto, a su vez, llevó a una discusión sobre el currículo de psicología, procurando aproximarla más a la realidad brasileña. Pero entonces se lograron resultados muy limitados: inserción de cursos electivos, disminución de la carga horaria total, mayor aceptación de las actividades de extensión, etc. Entre los cambios más importantes en la academia y la práctica psicológica, se registró una nueva perspectiva de la psicología (Lane \& Codo, 1984) que se plasmó en la creación de la Asociación Brasileña de Psicología Social (ABRAPSO). Esto ha permitido la acogida de enfoques argentinos (grupo operativo, psicología institucional), el construccionismo social, el pensamiento de Vigostky y la teoría de las representaciones sociales, abriendo un espacio de emergencia a multiplicidad de abordajes teóricometodológicos y prácticas aún vigentes.

Aunque, en la década de 1980 -y aún en la de 1990-, estos abordajes no obtenían financiamiento estatal porque los investigadores en psicología que eran evaluadores en las agencias financiadoras eran de orientación experimental y los consideraban "no científicos", en la actualidad hay un consenso de aceptación en la comunidad académica brasileña. Es decir, se ha producido un cambio de parámetros en las áreas de psicología de la CAPES y del CNPq. Sin embargo, como estas no son áreas autónomas, se mantienen en contacto/conflicto/diálogo con múltiples áreas, para establecer dichos criterios, la mayoría de las veces llegando a consensos que no atienden las prioridades de desarrollo de la psicología.

\section{Discusión}

Entre principios y mediados del siglo XX, en Argentina y Brasil, la psicología se ha desarrollado al amparo del Estado y en afinidad con sus intereses. Primero, en función de la moderna organización científica de sus instituciones, y más tarde, del desarrollo social en el contexto de procesos de expansión industrial y económica. En estas etapas, ambos países han contado con influencias teóricas similares que se recepcionaron por vías análogas. Incluso, han tenido en común la presencia de Emilio Mira y López que, en Argentina mediante sus escritos, colaboró en la difusión de la psicotecnia y en Brasil tuvo una decisiva actuación profesional, impulsando esta práctica y contribuyendo a la formalización de las carreras de psicología. En los dos países hubo abundantes instituciones psicotécnicas estatales y privadas, universitarias y extrauniversitarias, y fuerte apoyo estatal, pero en Argentina las que producían mayor investigación eran las universitarias estatales. En Brasil, por su parte, hubo una fuerte impronta de parte de extranjeros radicados en el país y de brasileños que estudiaron en el exterior antes de 1950.

Sin embargo, notamos las mayores diferencias entre la psicología de estos países, tras la profesionalización. En Argentina, desde la creación de la carrera de psicología, los grupos fundadores que traían la impronta psicotécnica o experimental, salvo excepciones, fueron quedando al margen de las mismas, ya sea por razones ideológicas, políticas o económicas. Estos exponentes emigraron o fueron insertándose en Facultades de Medicina, Ciencias Naturales o Arquitectura. Allí encontraron el apoyo presupuestario necesario, así como la apertura interdisciplinaria que los nutrió y ayudó a posicionarse internacionalmente. En dicho contexto, los investigadores que se fueron afianzando en el campo experimental o psicométrico tuvieron algún tipo de vínculo con CONICET (por medio de Houssay o Rimoldi), como becarios o investigadores, o conformando institutos dentro de su estructura con financiamiento más estable. Así se logró promover el avance de una psicología científica alternativa a la psicología perfilada en las carreras de psicología, de corte profesional y clínico, en un país en el que dicha ciencia no cuenta con la tradición de financiamiento privado. Esto ha tenido el costo de disociar la ciencia de la formación universitaria, con el progresivo vaciamiento de enfoques psicométricos, psicofísicos, psicobiológicos o experimentales y la reproducción de una matriz humanista y psicoanalista.

Como efecto social, la imagen del psicólogo ha sido predominantemente identificada con su rol clínico y psicopatológico, antes de 1984 disputado con los médicos. En suma, la psicología argentina ya no se asociaba a la producción de conocimiento 
útil para la modernización del Estado, como a principios del siglo XX, o el desarrollo socioeconómico del período 1945 y 1955. Desde mediados de 1960 hasta principios de 1980, solo una minoría de los psicólogos lograba desarrollar intereses compartidos y proyección conjunta con el Estado.

Por su parte, en Brasil, la formación del psicólogo tuvo que reglamentarse porque socialmente ya estaba instalada y se estaba institucionalizando como carrera primero desde la iniciativa privada, que fue alentada por el Estado, muy por el contrario de lo que ocurrió en Argentina. En efecto, la reglamentación de la carrera y la profesión del psicólogo en Brasil fueron consecuencia de un movimiento que, ya desde la década de 1950, contaba con numerosos profesionales actuando en el área, formándose mediante innumerables carreras de corta duración o autodidácticamente mediante la práctica profesional acompañada por grupos de estudio. Este movimiento fue capitalizado por asociaciones en las que estaban involucrados principalmente profesores universitarios. La impronta en la formación de todos era mayormente psicotécnica y experimental, aunque también tenían formación filosófica que les impedía el ostracismo.

La formación clínica también fue absorbida por las universidades, a pesar de que los psicoanalistas desarrollaran la suya propia, aun cuando desde los años ochenta con el advenimiento del lacanismo, estos buscaran ingresar en las instituciones universitarias y crear sus carreras de posgrado.

Respecto a los períodos posteriores a la creación de las carreras, si las dictaduras trajeron efectos sociales perniciosos en los dos países, es importante considerar que en Brasil no se apuntó a desmantelar la investigación en las universidades, sino a reordenarla y a financiarla en las mismas, fortaleciendo la ciencia psicológica y su producción en el ámbito estatal y privado, y planificando el perfil de graduado, así como evaluando los resultados del financiamiento.

Futuros estudios podrían analizar en qué medida la producción de ciencia psicológica y la formación científica se ha incorporado a la formación profesional del psicólogo, y cuál ha sido el papel de la psicología en la planificación estatal a partir de los procesos de recuperación democrática en nuestra región.

\section{Referencias}

Alzate-Medina, G. M. (2008). Efectos de la acreditación en el mejoramiento de la calidad de los programas de psicología en Colombia. Universitas Psychologica, 7(2), 425-439.

Antipoff, H. (1930). A psicologia na escola de aperfeiçoamento de Belo Horizonte. Archivos Brasileiros de Higiene Mental, 3(7), 226-240.

Antipoff, H. (1975). Teste MM. Belo Horizonte: Ibérica. (Originalmente publicado en 1943)

Antunes, M. A. M. (1999). A psicologia no Brasil-leitura histórica sobre sua constituição. São Paulo: Unimarco/Educ.

Ardila, R. (1979). La psicología en Argentina. Pasado, presente, futuro. Revista Latinoamericana de Psicología, 11(1), 71-91.

Ardila, R. (1986). La psicología en América Latina. Pasado, presente y futuro. México: Siglo XXI.

Baker, D., \& Benjamin, L. (2000). The affirmation of the scientist-practitioner. A look back at Boulder. American Psychologist, 55(2), 241-247.

Balán, J. (1991). Cuéntame tu vida. Una biografía colectiva del psicoanálisis argentino. Buenos Aires: Planeta.

Baptista, M. T. A. (2010). A regulamentação da profissão Psicologia: documentos que explicitam o processo histórico. Psicologia: Ciência e Profissão, 30(1), 170-191.

Barrionuevo, M., \& García Marcos, F. (1975). Carreras de psicología en universidades argentinas. Psicología Médica, 1, 84-110.

Bekerman, F. (2009). Investigación científica bajo el signo militar (1976-1983). La bisagra entre el CONICET y la universidad. Controversias y Concurrencias Latinoamericanas, 2(1), 189-206.

Boarini, M. L. (Ed.). (2012). Higiene mental: idéias que atravessaram o século XX. Maringá, PR: Editora da Universidade Estadual de Maringá.

Bomfim, E. (2001). Pierre Weil. En R. H. F. Campos (Coord.), Dicionário Biográfico da Psicologia no Brasil - Pioneiros. Rio de Janeiro: Imago. 
Brasil, Consejo Federal de Educación. Parecer 403 de 1962. Crea el currículo minima de las carreras de psicología.

Brasil, Decreto 981 de 1890. [Senado Federal]. Approva o Regulamento da Instrucção Primaria e Secundaria do Districto Federal. Noviembre 8 de 1890.

Brasil, Ley 4.119 de 1962. Dispõe sobre os cursos de formação em Psicologia e regulamenta a profissão de Psicólogo. Agosto 27 de 1962. DO 9253.

Brasil, Ley 5.540 de 1968. Fixa normas de organização e funcionamento do ensino superior e sua articulação com a escola média, e dá outras providências. Noviembre 28 de 1968. DO 10369.

Cabral, A. (2004). A psicologia no Brasil. En M. A. P. Antunes (Ed.), História da psicologia no Brasil: primeiros ensaios. Rio de Janeiro: EdUERJ. (Original publicado en 1950)

Calabresi, C., \& Polanco, F. (2011). La psicología y su enseñanza en Argentina. Entrevista con Hugo Klappenbach. Universitas Psychologica, 10(2), 613 626.

Campos, R. H. F. (2001a). Dicionário Biográfico da Psicologia no Brasil. Pioneiros. Rio de Janeiro: Imago.

Campos, R. H. F. (2001b). Helena Antipoff (1892-1974): A synthesis of Swiss and Soviet psychology in the context of Brazilian education. History of Psychology, 4(2), 133-158.

Carpintero, H. (2006). Horacio Rimoldi (1913-2006) y su significación en el marco de la psicología latinoamericana. Interdisciplinaria, 23(1), 7-16.

Carpintero, H., Ferrándiz, A., \& Lafuente, E. (1997). Juan Cuatrecasas y su visión psicológica de hombre. Cuadernos Argentinos de Historia de la Psicología, 3(1-2), 11-22.

Carrilho, H. (1925). Considerações geraes sobre prophylaxia mental e delinquencia. Arquivos Brasileiros de Higiene Mental, 1(1), 120-131.

Castro Solano, A. (2004). Las competencias profesionales del psicólogo y las necesidades de perfiles profesionales en los diferentes ámbitos laborales. Interdisciplinaria. Revista de Psicología y Ciencias Afines, 21(2), 117-152.

Centofanti, R. (1982). Radecki e a Psicologia no Brasil. Psicologia, Ciência e Profissão, 1, 3-50.
Centofanti, R. (2006). Os laboratórios de psicologia nas escolas normais de São Paulo: o despertar da psicometria. Psicologia Educacional, 22(1), 31-52.

Cirino, S. D., Miranda, R. L., \& Cruz, R. N. da (2012). The beginnings of behavior analysis laboratories in Brazil: A pedagogical view. History of Psychology, 15(3), 263-272.

Cirino, S. D., Miranda, R. L., \& de Souza, E. J., Jr. (2012). The laboratory of experimental psychology: Establishing a psychological community at a Brazilian university. Interamerican Journal of Psychology, 46(1), 135-142.

Coimbra, C. M. B. (1995). Guardiães da ordem: uma viagem pelas práticas psi na Brasil do Milagre. Rio de Janeiro: Autor.

Dagfal, A. (2009). Entre París y Buenos Aires. La invención del psicólogo (1942-1966). Buenos Aires: Paidós.

Da Matta, R. (1985). A casa e a rua. São Paulo: Brasiliense.

Detrixhe, M., \& Papini, M. (1978). Enrique Mouchet: 1886-1977. In memoriam. Revista Latinoamericana de Psicología, 10(2), 295-295.

Di Doménico, C., \& Vilanova, A. (Eds.). (1999). Formación de psicólogos en el Mercosur. Mar del Plata: Universidad Nacional de Mar del Plata.

Falcone, R. (2012). Psicología en Argentina: impronta europea y carácter nacional. Revista Interamericana de Psicología, 46(1), 87-98.

Ferraz, D. (2014). Memórias e histórias do curso de Psicologia da Faculdade Salesiana de Lorena/SP: uma contribuição para a historiografia da psicologia no Brasil. Tese de Doutorado. Programa de Pós-Gaduação em Psicologia Social. Uerj.

Ferrero, C., Iemme Khoury, V., \& Todisco, R. (2013). Antecedentes de investigación básica en Córdoba en el periodo 1960-1990 (Trabajo final de investigación para la obtención del título de Licenciado en Psicología). Facultad de Psicología, Universidad Nacional de Córdoba, Argentina.

Ferrero, L., \& Altamirano, P. (2011). Antecedentes de la psicología en Córdoba: demandas de los sectores industrial y educacional que propiciaron la formación de la carrera. Revista Tesis, 2, 3-20.

Figueira, S. (1985). Introdução: psicologismo, psicanálise e ciências sociais na "cultura psicanalítica". Emem 
S. Figueira (Ed.), Cultura da psicanálise (pp. 7-13). São Paulo: Brasiliense.

Frossard, N. C. (1930). Os test de Binet em nossos escolares. Arquivos Brasileiros de Higiene Mental, 3(4), 127-131.

Gallegos, M. (2010). La primera Conferencia Latinoamericana sobre entrenamiento en psicología (1974): el modelo latinoamericano y su significación histórica. Psicologia Ciencia e Profissao, 4, 792-809.

Gemelli, A. (1954). Le aporie della moderna psicologia. Humanitas, 1(3), 17-37.

Gentile, A. (2003). Ensayos históricos sobre psicoanálisis y psicología. Rosario: Fundación Ross.

Gonçalves, M. A. (2013). Uma análise das produções textuais Psicologia Social Comunitária no Brasil entre os anos 1990 e 2010 (Dissertação Mestrado em Psicologia). Universidade Federal do Rio de Janeiro, Brasil.

Gondra, J. G. (2004). Artes de civilizar: medicina, higiene e educação escolar na Corte Imperial. Rio de Janeiro: EdUerj.

Goulart, M. S. B., \& Santos, N. A. (2011). Departamento de Orientação e Treinamento do Banco da Lavoura de Minas Gerais. En A. M. Jacó-Vilela (Ed.), Dicionário Histórico de Instituições da Psicologia Brasileira. Rio de Janeiro: Imago.

Hereford, C. (1966). Current status of psychology in Latin America. Latin American Research Review, 1(2), 97-108.

Herschmann, M. M., \& Pereira, C. A. M. (Eds.). (1994) A invenção do Brasil moderno: medicina, educação e engenharia nos anos 20-30. Rio de Janeiro: Rocco.

Horas, P. (1949). Ambición y limitaciones del los test psicológicos. Revista de la Asociación de Empleados del Banco Mixto de San Luis, 2(6), 1-6.

Horas, P. (1961). La enseñanza de la psicología en la universidad argentina y otros comentarios. Anales del Instituto de Investigaciones Psicopedagógicas, 6, 339-354.

Jacó-Vilela, A. M. (1996). Livre como os pássaros, a formação do psicólogo (Tese de doutorado). Instituto de Psicologia, Universidade de São Paulo.

Jacó-Vilela, A. M. (2002). Idas e vindas do curso de psicologia no Brasil. Revista do Departamento de Psicologia, 1, 11-22.
Jacó-Vilela, A. M. (Ed.). (2011). Dicionário Histórico de Instituições da Psicologia no Brasil. Rio de Janeiro: Imago.

Jacó-Vilela, A. M. (2012). História da psicologia no Brasil. Uma narrativa pelo seu ensino. Psicologia: Ciência e Profissão, 32(Número Especial), 28-43.

Jacó-Vilela, A. M., Oliveira, F. M., Espírito Santo, A. A. do, Carneiro, F. D., Messias, M. C. N., \& Valente, N. F. (2007). Uma gestação silenciosa: a presença feminina nas instituições de psicologia no Rio de Janeiro na primeira metade do século XX. En M. A. T. Ribeiro \& J. Bernardes (Orgs.), A produção na diversidade: compromissos éticos e políticos em psicologia (Vol. 1, pp. 231-366). São Paulo: Casa do Psicólogo.

Klappenbach, H. (1996). Prólogo a la psicología experimental en la República Argentina de Horacio Piñero. Cuadernos Argentinos de Historia de la Psicología, 2(1-2), 239-269.

Klappenbach. H. (1999). La recepción orteguiana, Alberini y la renovación de la psicología argentina a partir de los veinte. Revista de Historia de la Psicología, 20(1), 87-95.

Klappenbach, H. (2001). Dos editoriales en los comienzos de la profesionalización de la psicología en Argentina. Memorandum, 1, 61-71.

Klappenbach, H. (2002a). La psicología en la Argentina en el período de entreguerras. Saber y Tiempo. Revista de Historia de la Ciencia, 13, 133-162.

Klappenbach, H. (2002b). Benjamín Aybar y el desarrollo de la psicotecnia y la orientación profesional. Epistemología e Historia de la Ciencia, 8(8), 183-189.

Klappenbach, H. (2003). La globalización y la enseñanza de la psicología en la Argentina. Psicologia em Estudo, 8(2), 3-18.

Klappenbach, H. (2005). Historia de la orientación profesional en Argentina. Orientación y Sociedad, $5,37-48$

Klappenbach, H. (2006). Periodización de la psicología en Argentina. Revista de Historia de la Psicología, 27(1), 109-164.

Klappenbach, H. (2009). Estudio bibliométrico de la Revista de Psicología de la Universidad Nacional de La Plata. 1964-1983. Revista de Psicología [Segunda época], 10, 13-65. 
Klappenbach, H. (2012). Informes sobre formación universitaria en psicología en Argentina: 1961-1975. Revista Interamericana de Psicología, 46(1), 181-192.

Klappenbach, H., \& Pavesi, P. (1994). Una historia de la psicología en Latinoamérica. Revista Latinoamericana de Psicología, 26(3), 445-481.

Kurowski, M. (2001). La obra psicológica de Juan Cuatrecasas Arumí (1899-1990). Madrid: Universidad Complutense de Madrid, Facultad de Psicología.

Lane, S. T. M. \& Codo, L. W. (1984). Psicologia Social: o homem em movimentos. São Paulo: Brasiliense.

Leme Lopes, M. B. (1930). Pesquisas sobre a memória de fixação. Archivos Brasileiros de Higiene Mental, 3(2), 235-240.

Leme Lopes, M. B., \& Fialho, I. A. (1929). Suggestões para o emprego dos test. Archivos Brasileiros de Hygiene Mental, 2(2), 68-67.

Lima, R. (2011). Clínica de Orientação Infantil (COI). En A. M. Jacó-Vilela (Ed.), Dicionário Histórico de Instituições de Psicologia no Brasil. Rio de Janeiro: Imago.

Lopes, E. (1931). Subsídio para a adaptação brasileira dos test de Binet-Terman. Archivos Brasileiros de Higiene Mental, 4(3), 180-207.

Lourenço Filho, M. B. (2002). Introdução ao estudo da Escova Nova. Rio de Janeiro: EdUERJ. (Original publicado en 1930)

Lourenço Filho, M. B.(2008). Teste ABC. Para a verificação da maturidade necessária à aprendizagem da leitura e da escrita (13.a ed.). Brasilia: Instituto Nacional de Estudos e Pesquisas Educacionais Anísio Teixeira. (Original publicado en 1928)

Mancebo, D. (1997). Da psicologia aplicada à institucionalização universitária: a regulamentação da psicologia enquanto profissão. Cadernos IPUB, 8, 161-177.

Massimi, M. (1990). História da psicologia Brasileira, da época colonial até 1934. São Paulo: E.P.U.

Massimi, M. (2006). Alimentos, palavras e saúde (da alma e do corpo), em sermões de pregadores brasileiros do século XVII. História, Ciências, Saúde, 13(2), 253-270.

Mikusinski, E., Horas, P., \& Gialluca, D. (octubre, 1969). Un programa de investigación psicosocial de la clase 1948. Trabajo presentado en el Primer Congreso Nacional de Psicología Social, Salta, Tucumán.
Moreira, J. (1925). A seleção individual de imigrantes no programma da hygiene mental. Archivos Brasileiros de Higiene Mental, 1(1), 70-85.

Motta, J. M. C. (2005). A psicologia e o mundo do trabalho no Brasil: relações, história e memória. São Paulo: Ágora.

Muñoz, M., \& Mañas, M. (2012). Investigación académica en Europa: la mirada de Francia. Entrevista a Juan Seguí. Diálogos, 3(21), 117-122.

Mustaca, A. (2006). La psicología científica y el análisis del comportamiento en Argentina. Avances en Psicología Latinoamericana, 24, 13-27.

Nóbrega, M. (2006). Diálogo sobre a conversação do Gentio. São Paulo: MetaLibri. (Original publicado em 1557)

Pantano Castillo, D. (1997). Inicios de la psicología en Argentina. Primer laboratorio de psicofisiología creado por Victor Mercante en San Juan, 1891. San Juan: Subsecretaría de Cultura/Ministerio de Desarrollo Humano/Gobierno de la Provincia de San Juan.

Papini, M. (1976). Datos para una historia de la psicología experimental argentina (hasta 1930). Revista Latinoamericana de Psicología, 8(2), 319-335.

Papini, M. (1978). La psicología experimental argentina durante el periodo 1930-1955. Revista Latinoamericana de Psicología, 10(2), 227-258.

Papini, M., \& Mustaca, A. (1979). La psicologia experimental argentina entre 1956 y 1978. Revista Latinoamericana de Psicología, 11(3), 349-361.

Penna, A. G. (1992). História da psicologia no Rio de Janeiro. Rio de Janeiro: Imago.

Pernambucano, U., \& Barreto, A. P. (1930). Ensaio de applicação do test das 100 questões de Ballard. Archivos Brasileiros de Higiene Mental, 3(9), 313-345.

Petersen, C. A. (2007). A historical look at psychology and the scientist practitioner model. American Behavioral Scientist, 50(6), 758-765.

Piñeda, M. A. (2005). El impacto de la psicología neoescolástica experimental en la psicología argentina a través de textos de psicología de circulación en el país: 1935-1965. Memorandum, 8(1), 88-105.

Piñeda, M. A. (2010). Inicios de la psicología como ciencia del comportamiento en San Luis (Argentina). Revista Argentina de Ciencia del Comportamiento, 2(1), 24-33. 
Piñeda, M. A. (2012). Psychology publications by professors at Argentine psychology programs: 1958-1982. Revista Interamericana de Psicología, 46(1), 111-122.

Plotkin, M. (2003). Freud en las Pampas. Buenos Aires: Sudamericana.

Radecki, W. (1929). Tratado de psicologia. Rio de Janeiro: Imprensa Militar.

Radecki, W., \& Radecka, H. (1925). Pesquizas experimentaes sobre a fadiga dos menores trabalhadores nas fábricas. Nota prévia. Archivos Brasileiros de Higiene Mental, 1(2), 181-184.

Ramos, A. (2001). O negro brasileiro, etnografia religiosa (5.a ed.). Rio de Janeiro: Graphia. (Original publicado en 1934)

Rimoldi, H. (1995). Testimonio autobiográfico. Cuadernos Argentinos de Historia de la Psicología, 1(1-2), 275-300.

Romero, R. (1926). Explicações indispensáveis. Prefácio aos vários escritos de Tobias Barreto de Menezes. En T. Barreto (Ed.), Obras completas (Vol. 10, p. XXIV, Edição do Estado de Sergipe). Rio de Janeiro: Pongetti. (Original publicado en 1900)

Rosas, P. (1997). Mira y Lopez e a psicologia aplicada no Brasil. Rio de Janeiro: Editorial da FGV.

Rose, N. (2008). Psicologia como uma ciência social. Psicologia 8 Sociedade, 20(2). Recuperado de http://www.scielo.br/scielo.php?pi$\mathrm{d}=$ S0102-71822008000200002\&script $=$ sci_arttext

Rossi, L. (1997). Entrevista al Profesor Jorge Bianchi. En L. Rossi (Ed.), La psicología antes de la profesión (pp. 166-173). Buenos Aires: Eudeba.

Rossi, L., Falcone, R., Kirsch, U., Rodríguez Sturla, P., Luque, E., Diamant, A., \& Sommer, V. (2001). Psicología: su inscripción universitaria como profesión. Buenos Aires: Eudeba.

Rossi, L., Ibarra, M. F., \& Kirsch, U. (2010). Psicología en Argentina: criterios psicológicos en los diseños de fichas del área laboral (1920-1945). Anuario de Investigaciones, 17, 401-407.

Roxo, H. B. B. (1900). Duração dos atos psíquicos elementares nos alienados (Tese de Doutoramento). Faculdade de Medicina, Rio de Janeiro.

Russo, J. A. (1993). O corpo contra a palavra: as terapias corporais no campo psicológico nos anos 80. Rio de Janeiro: UFRJ.
Saforcada, E. (1969). Problemas y riesgos de la psicología en Argentina. Revista de Psicología, 1, 49-55.

Santos, T. M. (1956). Noções de psicologia da criança. São Paulo: Companhia Editora Nacional.

Santos, T. M. (1958). Orientação psicológica da criança. São Paulo: Companhia Editora Nacional.

Santos, T. M. (1962). Noções de psicologia educacional. São Paulo: Companhia Editora Nacional.

Santos, T. M. (1963). Noções de psicologia da aprendizagem. São Paulo: Companhia Editora Nacional.

Santos, T. M. (1966). Noções de psicologia do adolescente. São Paulo: Companhia Editora Nacional.

Saviani, D. O. (2008). Legado educacional do regime militar. Campinas, 28(76), 291-312.

Scherman, P. (2009). De las prácticas experimentales de la psicología a la profesión de psicólogo. Universidad Nacional de Córdoba 1900-1960. Tesis de Doctorado no publicada. Córdoba: Universidad Nacional de Córdoba.

Sierra, J. C., \& Bermúdez, M. P. (2005). Hacia el título iberoamericano de psicología: análisis de los programas docentes de las carreras de psicología en Iberoamérica. Revista Mexicana de Psicología, 22, 224-242.

Talak, A. M., \& Courel, R. (2001). La formación académica y profesional del psicólogo en Argentina. En J. P. Toro \& J. Villegas (Eds.), Problemas centrales para la formación académica y el entrenamiento profesional del psicólogo en las Américas (pp. 2183). Buenos Aires: Sociedad Interamericana de Psicología.

Talak, A. M. (2000). Los primeros desarrollos académicos de la psicología en la Argentina: 1896-1919. En J. C. Ríos, R. Ruiz, J. C. Stagnaro \& P. Weissmann. Psiquiatría, psicología y psicoanálisis: historia y memoria (pp. 215-230). Buenos Aires: Polemos.

Tassara de Olivera, E. T., \& Bori, C. (1998). A abertura de novos espaços. Psicologia USP, 9(1), 153-154.

Triarhou, L., \& Del Cerro, M. (2006). Semicentennial tribute to the ingenious neurobiologist Christfried Jakob (1866-1956). European Neurology, 56, $176-188$

Velho, G. (1978). Duas categorias de acusação na cultura brasileira contemporânea. En S. Figueira (Org.), Sociedade e doença mental (pp. 54-64). Rio de Janeiro: Campus. 
Vezzetti, H. (1981). Locura y delito: la regeneración moral de los argentinos. Revista Argentina de Psicología, 30, 93-113.

Vezzetti, H. (1988). El nacimiento de la psicología en la Argentina. Buenos Aires: Puntosur.

Vezzetti, H. (1989). Freud en Buenos Aires: 1910-1939. Buenos Aires: Puntosur.

Vilanova, A. (2000). La formación académica del psicólogo en el mundo y en el país. En O. Calo \& A. M. Hermosilla (Eds.), Psicología, ética y profesión: aportes deontológicos para la integración de los psicólogos del Mercosur (pp. 107-118). Mar del Plata: Universidad Nacional de Mar del Plata.

Villaverde, A. (1954). Centros de psicología aplicada en la República Argentina. Humanitas, 1(3), 347-363.

Villegas, J. F., Marassi, P., \& Toro, J. P. (Eds.). (2003) Problemas centrales para la formación de los psicólogos en las Américas (Vol. 3). Santiago de Chile: Sociedad Interamericana de Psicología. 
